\title{
Docencia del ciberperiodismo: estrategias para una simulación eficaz
}

\author{
Emy ARMAÑANZAS SoduPE \\ Universidad del País Vasco \\ emiliana.armananzas@ehu.es
}

Recibido: $19 / 07 / 2012$

Aceptado: 30/10/2012

\begin{abstract}
Resumen
La implantación del Plan Bolonia, en el marco del Espacio Europeo de Educación Superior, supone una renovación de los planes de estudio de Periodismo, impartidos en las Facultades de Comunicación. Entre otros aspectos, se concreta en un reforzamiento de las asignaturas dedicadas al ciberperiodismo, entendido como el desarrollado por el periodista que trabaja en y para los medios online. Implica también una renovación pedagógica que aún muestra carencias. La rapidez de los cambios tecnológicos y de comportamientos sociales y profesionales del ciberperiodismo reportan un plus de complejidad-también de experimentación- para docentes y alumnos. Tratamos de identificar las dificultades y carencias comunes en la docencia del periodismo online con el fin de reflexionar y proponer estrategias que lleven a una mayor aproximación de las aulas a la profesión ciberperiodística.
\end{abstract}

Palabras clave: Docencia online, ciberperiodismo.

\section{Teaching of online journalism: strategies for effective simulation}

\begin{abstract}
The implementation of the Bologna Process (European Area of Higher Education) is a renewal of Journalism curriculum, taught in the Faculty of Communication. Among other aspects, in particular the strengthening of the subjects engaged in online journalism, understood as that developed by the journalist working in the online media. It also implies a pedagogical renewal that still shows weaknesses. The speed of technological, social behaviors and online journalism changes add complexity -also experimentation -for teachers and students. We try to identify common problems and shortcomings in teaching online journalism al University to reflect and propose strategies that lead to greater approximation of online journalism profession.
\end{abstract}

Key words: Teaching online journalism

\section{Referencia normalizada}

ARMAÑANZAS SODUPE, Emy (2012): "Docencia del ciberperiodismo: estrategias para una simulación eficaz”. Estudios sobre el mensaje periodístico. Vol. 18, núm. especial octubre, págs.: 77-85. Madrid, Servicio de Publicaciones de la Universidad Complutense.

Sumario: 1. Introducción. 2. Metodología. 3. Dificultades y carencias de la docencia del ciberperiodismo; 3.1. Materias escasas y optativas; 3.2. Poca dotación tecnológica; 3.3. Contraste generacional. 4. Estrategias para la nueva docencia del ciberperiodismo; 4.1. Tarea colectiva y esfuerzo individual; 4.2. Pedagogía renovada; 4.3; Simular el trabajo del ciberperiodista; 4.4. Univesidad y cibermedios. 5. Conclusiones. 6 . Referencias bibliográficas.

\section{Introducción}

La finalidad de la docencia del ciberperiodismo es la de preparar al alumno para que sea un profesional del periodismo online y pueda trabajar en el nuevo escenario laboral que demanda nuevos perfiles profesionales y muchas más posibilidades de trabajo y de negocio, pero que también plantea una competencia más intensa al vivir en una sociedad global. 
El Plan Bolonia supone un esfuerzo tecnológico a través de la aplicación de las Tecnologías de la Información y de la Comunicación (TIC) en la docencia, con una intensificación del lado práctico de las materias así como la modificación de los usos pedagógicos. Cambios vistos aún como insuficientes para alcanzar el modelo de Escuela Digital (Martín Bernal, 2009; Sigalés et al, 2009; Benito, 2009; Marchesi y Díaz, 2009; Segura, 2009).

En los estudios universitarios del periodismo online se aprecian esfuerzos que avanzan en un sentido positivo, si bien con muchas dificultades tanto en el plano tecnológico, de las materias, del docente y del destinatario del mismo, el alumno (Tejedor, 2008; Aguaded Gómez et al, 2010). No es un secreto para nadie que la constante renovación tecnológica de los cibermedios y los usos facilitados por esa tecnología corren más que los cambios docentes y su adaptación a la enseñanza/aprendizaje (Armañanzas, 2010a).

En el aula, primero el docente y luego el alumno tienen que dominar el rol del periodista online y las TIC en las que se apoya su trabajo, a través de una buena base teórica y de la práctica de las futuras funciones que desarrollarán en la Red así como de los dispositivos que tendrán que aprender a manejar con la finalidad de simular su futuro trabajo en los cibermedios.

Sabemos que los cambios docentes encuentran dificultades que ralentizan su progresión, bien del lado tecnológico o del humano; todas deberían resolverse cuanto antes. Tratamos aquí de identificar carencias y dificultades comunes con el fin de proponer estrategias que aproximen las aulas a la profesión ciberperiodística (Fumero y Roca, 2007).

\section{Metodología}

Repasamos el estado de los estudios de ciberperiodismo en nuestras Facultades de Comunicación antes y después de los cambios introducidos por el Plan Bolonia. Se han considerado las distintas experiencias docentes en asignaturas que atañen al periodismo online, publicadas por profesores tanto en libros como en artículos analógicos y digitales. También se tienen en cuenta las publicaciones sobre recursos docentes aplicados a la enseñanza del ciberperiodismo. Asimismo, se referencian las experiencias y conclusiones expuestas en congresos sobre ciberperiodismo, así como textos periodísticos que dan a conocer, antes que las revistas académicas, los nuevos dispositivos y sus aplicaciones ciberperiodísticas.

\section{Dificultades y carencias de la docencia del ciberperiodismo}

\subsection{Materias escasas y optativas}

La incorporación del ciberperiodismo a los planes de estudio de las Facultades de Comunicación se encontraba aún en fase inicial diez años después de que Internet hubiera comenzado a introducirse en España y los primeros periódicos colgaran en la Red sus ediciones electrónicas (Armañanzas et al, 1996). No fue consecuencia de un plan coherente sino producto espontáneo de la voluntad de profesores que vieron necesaria la incorporación de la enseñanza del periodismo digital. 
Solo 17 materias, el 7\% del total de las ofertadas en la licenciatura de Periodismo en 26 Universidades españolas, se dedicaban a la enseñanza del periodismo online de manera exclusiva. En un $40 \%$ eran optativas, con escasa bibliografía y materiales multimedia, con el principal objetivo del estudio de la estructura de medios digitales. Las prácticas apenas incidían en la creación de materiales y proyectos multimedia informativos (Tejedor, 2008).

El reciente Plan Bolonia incluye nuevas asignaturas obligatorias de ciberperiodismo en todos los cursos, con recorrido curricular como la web 2.0, la tele y la radio online, $\mathrm{y}$ temas relacionados con el periodismo digital en todo el conjunto de materias de Periodismo. Otras mejoras suponen menos estudiantes en los grupos, más trabajo en equipo, desarrollo de proyectos y el uso de herramientas de la información y la comunicación lo que favorece su docencia.

Las TIC son imprescindibles para la enseñanza/aprendizaje del ciberperiodismo, de ahí la importancia de que el centro cuente con ellas y el profesor las conozca. Sin olvidar los conocimientos teóricos (conceptos abstractos, complejos) en torno al periodismo online y a otras materias que harán del periodista un profesional con mayor cultura y criterio. Pero falta la "articulación de un corpus de fundamentos teóricos que sustente una práctica fehacientemente renovadora" (Martín Bernal, 2009).

Una situación que se agrava ya que el alumno sabe usar las nuevas tecnologías en el ámbito particular pero, en general, presenta notables carencias para su posterior proyección laboral. Su comprensión lectora, el manejo del lenguaje oral y escrito, la capacidad de razonamiento, de análisis y síntesis dejan mucho que desear.

\subsection{Poca dotación tecnológica}

Para que esta renovación docente cuaje necesita tiempo y aunar esfuerzos tanto tecnológicos como pedagógicos ya que presentan carencias. Los centros docentes y los de investigación están aún poco dotados tecnológicamente. Y no se da en ellos una verdadera relación entre los adelantos técnicos y su aplicación real en la creación de nuevos modelos de difusión, de conservación, de conocimiento (Lucía, 2012).

No ha existido un plan general de la aplicación de las TIC a la enseñanza universitaria en Comunicación aunque se hayan instalado la Red y los equipos. Tampoco uno de asistencia técnica, ampliación y renovación de equipos y de software que pronto quedan obsoletos. Hay que exigir a la universidad más técnica pero con el inconveniente de la limitada movilidad que permiten los ordenadores de sobremesa. Incluso habría que cuestionar el concepto de aulas de informática ya que la tecnología tiene que encontrarse a disposición del docente cuando considere necesario incorporarla a la práctica hasta hacerse "invisible" (Aguaded et al, 2010: 65).

\subsection{Contraste generacional}

Las TIC forman parte de la vida diaria de docentes y alumnos, pero se trata de que los profesores enseñen su aplicación al ciberperiodismo. En el aula, los docentes se encuentran ante un profundo cambio generacional en el entorno tecnológico ya que no todos están formados en dicha tecnología y en sus aplicaciones. Serían "inmigrantes digitales", como los que no han utilizado las TIC desde temprana edad (Prensky, 2001 y 2004), mientras que sus alumnos son, en parte, "nativos digitales". 
Para salvar esta distancia generacional no se ha contado con un diseño previo, institucional y unificado de formación de un profesorado que va a desempeñar nuevos roles pedagógicos; más bien se ha dejado a su decisión personal. Además, muchos profesores no han ejercido de periodistas y si lo han hecho, no como ciberperiodistas. También desconocen otros idiomas, como el inglés, fundamental en la Red para abordar multitud de contenidos. Por lo que existe un "desfase -o más bien rotunda disparidad- entre la potencialidad de las TIC incorporadas a las aulas y la flaca renovación de los procesos pedagógicos" (Martín Bernal, 2009).

Ante este profesor se encuentran en el aula estudiantes desiguales respecto a la práctica digital. Son "nativos digitales" en el uso cotidiano de la Red, que les pone en contacto con las comunidades de usuarios con intereses comunes (blogs, foros, chats, redes sociales), en el intercambio de mensajes, información y archivos multimedia; en la lectura interactiva y los videojuegos. Y son inmigrantes por su falta de conocimiento de los usos técnicos para desarrollar el trabajo del ciberperiodista así como de conceptos teóricos y de otros idiomas, como el inglés.

Todo ello dificulta la enseñanza/aprendizaje del ciberperiodismo pero las oportunidades se abren enormemente para profesores, investigadores y alumnos aunque el camino sea complejo. Al docente se le pide preparación en su materia y en recursos digitales, con lo que habrá que redefinirlo. Al alumno, que no se distraiga con la mera técnica, con la sola información, sino que todos estos valiosos recursos digitales le hagan llegar al conocimiento. A ambos, que estén abiertos a la renovación pedagógica. Decía Albert Einstein que si pretendes resultados diferentes no puedes hacer siempre lo mismo. De ahí que haya que plantear nuevas estrategias docentes.

\section{Estrategias para la nueva docencia del ciberperiodismo}

Pese a las carencias y dificultades, la enseñanza/aprendizaje del ciberperiodismo crece y debe ir mejorando. Hay autores que posan la evolución hacia la educación online en la capacidad innovadora de los nuevos usuarios que empujarán hacia la renovación de los modelos pedagógicos y del diseño de herramientas, sistemas y servicios (Fumero y Roca, 2007). Otros opinan que son las instituciones políticas y educativas las que tienen la principal responsabilidad de la renovación pedagógica a través de la planificación y actuación así como proporcionando recursos (Martín Bernal, 2009).

Ambos planteamientos ya se están dando aunque los institucionales se quedan cortos, sobre todo en tecnología y preparación de los profesores. Y son mejorables los relativos a los docentes y alumnos y a la aproximación de ambos a las prácticas de los medios online. La tecnología es la que no deja de evolucionar hacia nuevos usos comunicativos que todos acabaremos aprendiendo y utilizando. La dificultad se acrecienta porque esta evolución técnica y su uso en la sociedad es tan rápida que enseñantes y alumnos nos convertimos en lentos y mostramos carencias en su docencia/aprendizaje.

\subsection{Tarea colectiva y esfuerzo individual}

Por esta razón es tan importante crear una red de conocimiento teórico y de experiencias sobre ciberperiodismo ya que la tarea para un solo profesor es inabordable. Los docentes deben aunar esfuerzos para desarrollar nuevos materiales adaptados a las 
asignaturas de los estudios de Comunicación abiertos a todos de distintas universidades y países accediendo a los repositorios centralizados y actualizados de software. Lo que se completaría con la creación de observatorios que velen por el análisis y el seguimiento de los avances sobre ciberperiodístico, con el desarrollo bibliográfico y de proyectos de investigación, máster, doctorados y foros de debate (Tejedor, 2008: 37 y 38).

La utilización de plataformas digitales, llamadas "plataformas de conocimiento" (Lucía, 2012: 119-133), en la docencia universitaria ha ido creciendo. Entre los recursos digitales que ofrecen, los de información y comunicación (programas de asignaturas, propuestas de prácticas y actividades, documentación, artículos, informes, bibliografía, etc.) para la asimilación de contenidos son los más utilizados por los profesores (Aguaded et al, 2010).

Pero tienen que crecer los recursos para la generación de conocimiento (blogs, wikis, tesauros, bitácoras, materiales multimedia, etc.), de carácter cooperativo, basados en la actividad del estudiante (prácticas con software específico, tutorías grupales, lectura y comentario de documentos, proyectos de trabajos, presentaciones multimedia, materiales audiovisuales e interactivos) que son los menos frecuentados por los docentes, según el autor.

Hasta que no avancen más los sistemas de producción de materiales didácticos con aporte de las TIC para todos, el docente tendrá que preparar individualmente los suyos adaptados a los nuevos soportes (páginas web, WebQuest, material multimedia, etc.). Para ello, necesita formación continua y reciclaje en las aplicaciones y usos de las TIC en el plano comunicativo, tanto si imparte materias de ciberperiodismo como si son asignaturas con una vinculación menos directa con la Red.

La inclusión de las TIC en la enseñanza es una innovación curricular con grandes posibilidades didácticas al suponer la revisión y modificación de los objetivos, de los contenidos, del método didáctico, la exploración de nuevos caminos, cambios en el aula y en la evaluación de la asignatura que pasa a ser continuada. No se debe enseñar de la misma manera a unos alumnos que ya no son iguales: tienen otros hábitos, se relacionan diferente, no leen ni escriben igual ni van a aprender como siempre (Armañanzas, 2000).

\subsection{Pedagogía renovada}

Los nuevos estudios de grado tienen el objetivo de equilibrar la teoría y la práctica (Haug, 2008). Es decir, que el carácter formativo del pensamiento comparta espacio con los contenidos informativos prácticos con el fin de llegar al conocimiento. Se trata de la enseñanza/aprendizaje de las materias que posibiliten al estudiante gestionar la información en su ámbito de búsqueda y verificación, valoración, organización y distribución de datos. También la creación de nuevos contenidos interactivos, hipertextuales, con narración multimedia, así como conocer la Red y los recursos técnicos y dominar la comunicación con los usuarios, cada vez más importante. En definitiva, un aprendizaje para los futuros ciberperiodistas de mayor alcance, profundidad y creatividad a través de una constante revisión de los planes estudio.

No solo varían las materias de estudio sino su pedagogía. Hasta ahora, el profesor lo aportaba todo: los datos, el camino a recorrer, la bibliografía. Con las TIC, el alumno 
tiene que hacerse con la información que luego aprovechará y analizará en unos trabajos apoyados también por los medios técnicos precisos. El esfuerzo del alumno será mayor, tomará más decisiones pero estará más motivado por la diversidad de medios: vídeos, gráficos, sonido, música, fotografía, textos que facilitan la asimilación de conocimientos. El resultado será más gratificante al ser un trabajo más creativo y reflexivo.

Se genera mayor participación en las clases permitiendo el aprendizaje en grupo y también de manera autónoma. El profesor pasa a ser un guía que sugiere a los alumnos. Su papel se intensifica y se hace más creativo ya que es quien dinamiza, organiza y elabora su propia manera de enseñar. En vez de preparar un circuito común para el colectivo de alumnos, tendrá que hacer un seguimiento personalizado de cada estudiante con el fin de valorarle no solo los resultados sino su proceso de aprendizaje.

\subsection{Simular el trabajo del ciberperiodista}

El profesor es el responsable de que la tecnología no se quede en mera acumulación de información sino que ayude a la asimilación y comprensión de conceptos y procedimientos para la construcción de conocimiento. Para ello, la Red y su dimensión de web 2.0. es el ámbito idóneo para que el docente prepare materiales teóricos y prácticos y para que el alumno practique las habilidades digitales aplicándolas al ciberperiodismo.

Desde los emails y envío de archivos al contacto digital con comunidades de usuarios a través de blogs, foros, chats para crear grupos de discusión y wikis sobre materias de estudio. Asimismo, la búsqueda y verificación de documentación (artículos, ebooks, imágenes, documentos sonoros) relacionada con el ciberperiodismo a través de diversas fuentes (bibliotecas, informes, medios de comunicación, etc.).

Se trata de simular en el aula lo más fielmente posible el trabajo del ciberperiodista a través de la enseñanza de las materias vinculadas al mismo. Un profesional que domina la creación de mensajes periodísticos multimedia, las bases de datos en Red, la creación de webs y blogs personales, que puede fotografiar, diseñar, editar, documentar. El docente, entonces, debe conocer y enseñar a sus alumnos la estructura de los cibermedios, el comportamiento de las audiencias en las redes sociales, los blogs de periodistas, las fuentes y webs de contenido periodístico así como los dispositivos, tan frecuentemente renovados, que se utilizan en los cibermedios.

\subsection{Universidad y cibermedios}

El ordenador de sobremesa ya no es el dispositivo inteligente más vendido: la evolución camina hacia los portátiles, los smartphones, tabletas digitales, netboooks, ereaders, PDA, etc. (Fundación Telefónica, 2012: 50; Jiménez de Luis, 2012). Se necesitan más inversiones en nuevos dispositivos pero estamos en tiempos de ahorro. Por ello exponemos dos propuestas de las que se beneficiarían las universidades, las empresas de tecnología y de medios de comunicación. Una de ellas consistiría en que los centros docentes acordaran con las empresas de tecnología compras muy ventajosas económicamente y préstamos de uso de dispositivos de última generación en las aulas. 
Su manejo y prestaciones deberían conocerlos los alumnos ya que el ordenador solo es un dispositivo más de los muchos que utilizarán en su trabajo. Mientras que aquí aún hacemos el camino del e-learning, las universidades norteamericanas y canadienses, fundamentalmente, y las escuelas de negocios aplican la metodología mobile learning. Es decir, uso de dispositivos que permiten la conexión y el acceso a la información en cualquier lugar y en todo momento (Armañanzas, 2010b). Algo que también han introducido los cibermedios para que sus profesionales trabajen y transmitan información online estén donde estén.

En contrapartida beneficiosa para las empresas, los alumnos estarán aprendiendo a utilizar dispositivos tecnológicos de unas determinadas marcas comerciales y modelos que serán los que acaben manejando mejor y, probablemente, los que compren. Para poder enseñar a obtener las mejores prestaciones de estos dispositivos que se renuevan constantemente y cuyas aplicaciones son imprescindibles para los ciberperiodistas, antes los profesores tendrán que saber manejarlos. Pero no son suficientes los cursos y la parte autodidacta que el docente pone en juego para ese fin sino que debería aprender su uso en las empresas de comunicación. Esta es la segunda propuesta.

La mayoría de los docentes han trabajado exclusivamente en la universidad pero están enseñando en las aulas a futuros periodistas online. Por ello, deberían pasar cierto tiempo en las empresas de cibermedios con la finalidad de ponerse al día en las aplicaciones a funciones concretas (géneros, fuentes, usos de webs, búsqueda y creación de información, contacto permanente entre periodistas y usuarios, etc.) de esos dispositivos. Estos convenios entre universidades y cibermedios les servirían a las empresas para promocionar sus cabeceras entre los alumnos ya que los profesores pondrán ejemplos concretos de lo que han vivido en esas empresas mediáticas.

Los docentes podrán aportar a los ciberperiodistas conocimientos teóricos, investigaciones, estudios comparativos de cualquier país ya que en las redacciones se deja de leer teoría periodística por lo acuciante del trabajo diario. Asimismo, las empresas podrán encargar a los docentes estudios de su interés. Y entre todos se podrían organizar foros de debate, seminarios, intervenciones de profesionales del ciberperiodismo en los máster universitarios; intercambiar iniciativas y proyectos que enriquecerían tanto a la universidad como a los medios de comunicación.

\section{Conclusiones}

1. El Plan Bolonia favorece la docencia/aprendizaje del ciberperiodismo, pero muestra aún carencias en cuanto a las materias, la tecnología, los docentes y la pedagogía.

2. Las estrategias para la nueva docencia del periodismo online deben basarse en un esfuerzo colectivo e individual de profesores y alumnos que introduzca renovados métodos pedagógicos de enseñanza y aprendizaje. Asimismo, que cuenten con la Red como ámbito preferente y que crezca en los recursos para la generación de conocimiento: creación de blogs, wikis, tesauros, bitácoras, materiales multimedia, entre otros.

3. Asimismo es necesario simular el trabajo del ciberperiodista en el aula, en la Red y a través de convenios con los cibermedios para profesores y alumnos. 
4. La revisión de los planes de estudio del periodismo online debe ser constante, dada la rapidez de los cambios técnicos y sus aplicaciones.

\section{Referencias bibliográficas}

AGUADED GÓMEZ, José Ignacio et al (2010): "Estudio sobre el uso e integración de plataformas de teleformación en universidades andaluzas", en Didáctica, Innovación y Multimedia: http://www.erevistas.csic.es/ficha articulo.php?url =oai:raco.cat:article/214693\&oai_iden=oai_revista192. [fecha de consulta: $17 \mathrm{de}$ septiembre de 2012]

ARMAÑANZAS, Emy et al. (1996): El periodismo electrónico. Información y servicios multimedia en la era del ciberespacio. Barcelona, Ariel.

ARMAÑANZAS, Emy (2000): "El libro electrónico: Un hito cultural y comercial”, en VV.AA.: El bienestar en la cultura. Bilbao, Universidad del País Vasco, pp. 77-97.

ARMAÑANZAS, Emy (2010a): "El libro electrónico, una gran herramienta para la cultura y la educación", en Actas del Congreso Euro-Iberoamericano. Alfabetización mediática y culturas digitales: http://www.gabinetecomunicacionyeducacion.com/files/adjuntos/El\%20libro\%20electrónico,\%20una\%20gran\%20herramie nta\%20para\%20la\%20educación.pdf [fecha de consulta: 5 de julio de 2012]

ARMAÑANZAS, Emy (2010b): "La revolución del libro multimedia", en Actas del XXV Congreso Internacional de Comunicación "Modelos de negocio para una economía digital: El valor de los contenidos": http://www.unav.es/comunicacion/cicom /ediciones.../CICOMXXVResumenes.pdf. [fecha de consulta: 5 de julio de 2012]

BENITO, Manuel (2009): "Desafíos pedagógicos de la escuela virtual. Las TIC y los nuevos paradigmas educativos", en Telos. Cuadernos de Comunicación e Innovación: http://sociedadinformacion.fundacion.telefonica.com /telos/articulocuaderno.asp@idarticulo=2\&rev=78.htm [fecha de consulta: 16 de septiembre de 2012]

FUMERO, Antonio y ROCA, Genís (2007): Web 2.0. Madrid, Fundación Orange.

FUNDACIÓN TELEFÓNICA (2012): La sociedad de la Información en España 2011. Madrid, Ariel y Fundación Telefónica.

HAUG, Guy (2008): "Legislación europea y legislaciones nacionales". Revista de Educación, $\mathrm{n}^{\circ}$ extraordinario. Madrid, Ministerio de Educación, Cultura y Deporte, pp. 285-305.

JIMÉNEZ DE LUIS, Ángel (2012): "El ipad encoge para entrar en empresas y escuelas", en El Mundo, 24 de octubre, sección Comunicación, p. 64.

LUCÍA, Juan Manuel (2012): Elogio del texto digital. Claves para interpretar el nuevo paradigma. Madrid, Fórcola.

MARCHESI, Álvaro y DÍAZ, Tamara (2009): "Desafíos de las TIC. El cambio educativo en Iberoamérica", en Telos. Cuadernos de Comunicación e Innovación: http://sociedadinformacion.fundacion.telefonica.com/telos/articulocuaderno.asp@i darticulo $=6 \&$ rev $=78 . \mathrm{htm}$ [fecha de consulta: 16 de septiembre de 2012]. 
MARTÍN BERNAL, Obdulio (2009): "Educación 2.0. Horizontes de la innovación en la escuela", en Telos. Cuadernos de Comunicación e Innovación: http://sociedadinformacion.fundacion.telefonica.com/telos/articulocuaderno.asp@idarticulo $=1 \&$ rev $=78 . \mathrm{htm}$ [fecha de consulta: 18 de septiembre de 2012]

PRENSKY, Marc (2001): “Digital Natives, Digital Immigrants", en On the Horizon: http://www.marcprensky.com/writing/Prensky\%20\%20Digital\%20Natives, $\% 20$ Digital $\% 20$ Immigrants $\% 20 \% 20$ Part 1.pdf [fecha de consulta: 5 de julio de 2012]

PRENSKY, Marc (2004): "The Emerging Online Life of the Digital Native: What they do differently because of technology, and how they do it", en Work in progress: http://www.marcprensky.com/writing/PrenskyThe_Emerging_Online_Life_of_the_Digital_Native-03.pdf. [fecha de consulta: 5 de julio de 2012]

SEGURA, Mariano (2009): "Panorama internacional de las TIC en la Educación. Barreras actuales y propuestas de futuro", en Telos. Cuadernos de Comunicación e Innovación: $\quad \mathrm{http}: / /$ sociedadinformacion.fundacion.telefonica.com/telos /articulocuaderno.asp@idarticulo=3\&rev=78.htm [fecha de consulta: 17 de septiembre de 2012]

SIGALÉS, Carles et al (2009): "TIC e innovación en la educación escolar española. Estado y perspectivas", en Telos. Cuadernos de Comunicación e Innovación: http://sociedadinformacion.fundacion.telefonica.com/telos/articulocuaderno.asp@i darticulo $=4 \&$ rev $=78 . h t m$ [fecha de consulta: 18 de septiembre de 2012]

TEJEDOR, Santiago (2008): “Ciberperiodismo y universidad: diagnósticos y retos de la enseñanza del periodismo on-line". Anàlisi. Quaderns de comunicació i cultura,

\section{Emy ARMAÑANZAS SODUPE}

Profesora titular

Universidad del País Vasco. Bilbao

emiliana.armananzas@ehu.es

Alameda de Mazarredo ${ }^{\circ} 18,6^{\circ}$

Bilbao 48009 